\title{
Circulating PAMM, a novel antioxidant and anti-inflammatory protein, is elevated in acute $\mathrm{SCl}$
}

\author{
Leslie R. Morse, Nguyen Nguyen, Yan Xu, Prakash Jha and Ricardo A. Battaglino*
}

\begin{abstract}
Background: Peroxiredoxin activated in M-CSF stimulated monocytes (PAMM) is a novel protein produced by adipocytes with putative redox regulatory and anti-inflammatory properties. Because acute spinal cord injury (SCI) is associated with oxidative stress and neuroinflammation and because PAMM can be detected in systemic circulation, we hypothesized that acute neuro-trauma might induce changes in circulating PAMM expression. Specifically, we hypothesized that PAMM levels might vary based on the presence or absence of acute, traumatic SCI. We therefore investigated circulating PAMM levels in adults with and without acute traumatic SCl.

Methods: We studied 105 men and women (54 with SCI and 51 without SCI). Participants with SCI were admitted for acute rehabilitation within 1 month after injury. Serum samples were obtained during hospitalization and stored at $-80^{\circ} \mathrm{C}$ until batch analysis. Total PAMM was quantified by ELISA assay (MyBiosource, Cat. No: MBS9327247) with a detection limit of $0.25 \mathrm{ng} / \mathrm{ml}$. Separate multivariate models including age, BMl, and injury severity were assessed to determine significant clinical predictors of change in PAMM levels.
\end{abstract}

Results: When adjusting for BMI, age, and gender, mean change in PAMM levels were greatest in participants with motor complete $\mathrm{SCl}$ compared to able-bodied $(1.65 \mathrm{ng} / \mathrm{ml}$ versus $0.94 \mathrm{ng} / \mathrm{ml}, \mathrm{p}=0.003)$. This model explained $26 \%$ of the variation in change in circulating PAMM levels.

Conclusions: Our results suggest that PAMM may be a novel biomarker of neurological injury or of native antiinflammatory responses to neurological injury. More work is needed to establish the role of PAMM and other adipocyte-derived factors in the acute response to neurotrauma.

Keywords: PAMM, Biomarker, Inflammation, Reactive oxygen species, Spinal cord injury, Rehabilitation medicine

\section{Background}

According to the International Spinal Cord Society Prevention Committee, the global-incident rate of traumatic Spinal Cord Injury (SCI) is 23 cases per million [1]. SCI results in varying degrees of functional impairment based on the extent and location of trauma to the cord with high cost to both individual and society. The assessment of

*Correspondence: rbattagl@umn.edu

Department of Rehabilitation Medicine, University of Minnesota School of Medicine, 500 Boynton Health Service Bridge, 410 Church St. SE, Minneapolis, MN 55455, USA both injury severity and long-term neurological prognosis remain challenging after SCI [2]. While injury severity is routinely determined by neurological exam, there are instances where complete neurological testing cannot be performed (coma, intubated or nonverbal patient, presence of cast or other physical barrier). Furthermore, there is substantial variability in spontaneous neurologic recovery at 1-year post-injury and neurological examination does not differentiate between those who will experience recovery from those who will not. This variability in spontaneous recovery necessitates very large numbers for clinical trials that test novel neuroprotective therapies 
acutely after injury. There are no validated biomarkers of injury severity, neurological recovery, or response to neuroprotective therapy and this is considered a major limitation to both clinical care and to the development of adequately designed and powered clinical trials. Identification of a novel biomarker of spontaneous recovery would make clinical trial recruitment more economical and efficient. Identification of a biomarker that is easy to obtain and measure (i.e. circulates in blood) would represent a major advance in the field.

Trauma results in primary injury due to mechanical forces leading to compression or contusion of the cord. Subsequently, secondary injury occurs via multiple mechanisms including reactive oxygen/nitrogen species (ROS/RNS) generation, glutamate-mediated excitotoxicity, cellular necrosis and apoptosis [3-5], and the development of neuroinflammation. Oxidative stress occurs in primary injury due to cellular releases of cytoplasmic components and mitochondrial dysfunction and persists through the secondary injury phase due to severe neuroinflammation [5]. We recently identified a novel secreted protein with both antioxidant and redox-regulating activity which we called Peroxiredoxin-like 2 activated in M-CSF stimulated monocytes (PAMM) [6, 7], also known as adiporedoxin (Adrx) [8]. PAMM is broadly expressed across tissues, but is most highly expressed in white and brown adipose tissue. Furthermore, we demonstrated anti-oxidant and anti-inflammatory properties of PAMM in both in vitro and rodent in vivo models. Based on these properties, and because acute $\mathrm{SCI}$ is associated with neuroinflammation and can be detected in systemic circulation, we hypothesized that acute neurotrauma might induce changes in circulating PAMM expression. We furthermore hypothesized that PAMM is a putative biomarker of acute SCI. We therefore investigated circulating PAMM levels in men and women with and without acute spinal cord injury.

\section{Materials and methods Subjects}

We studied participants with traumatic SCI enrolled in our de-identified SCI biorepository. Participants were eligible for inclusion in the biorepository if they were 16 years or older, were inpatients receiving care for SCI at our rehabilitation hospital, and if they underwent a clinical blood draw during their inpatient stay. Serum samples were collected as discarded medical material at the time of each clinical draw obtained during the course of the rehabilitation stay, processed by the clinical laboratory, and banked for batch analysis. We collected samples from 141 individuals between December 10, 2012 and September 30, 2013. For the current analysis we selected for analysis a convenience sample of 54 adults with acute traumatic SCI. 51 age- and gender-matched men and women without SCI were studied as uninjured controls. The participants without SCI reported no medical conditions and were not taking medications at the time of study. Our Institutional Review Board approved this study.

\section{Variable definition}

Clinical and demographic information including age, gender, motor level and completeness of SCI, medical history, date of clinical draw, height, and weight were obtained by medical record review at the time of enrollment and recorded in a de-identified fashion. Age and body mass index (BMI) were considered as continuous variables. SCI severity was considered in two categories: motor complete (AIS A/B) and motor incomplete (AIS C/D).

\section{Biochemical analyses}

Plasma samples were drawn into an EDTA tube and immediately delivered to the core blood research laboratory at our facility. The samples were centrifuged for $15 \mathrm{~min}$ at $2600 \mathrm{rpm}(1459 \times g)$ at $4{ }^{\circ} \mathrm{C}$ and stored at $-80{ }^{\circ} \mathrm{C}$ until batch analysis. Total PAMM was quantified by ELISA assay (MyBiosource, Cat. No: MBS9327247) with a detection limit of $0.25 \mathrm{ng} / \mathrm{ml}$ and an intra-and inter-assay $\mathrm{CV}$ of less than $15 \%$ as previously described [8].

\section{Statistical analysis}

We used univariate and multivariable regression models to assess associations between clinical variables and circulating PAMM levels. Factors with a p-value of $<0.10$ in the univariate models, as well as factors that were deemed clinically significant (age, gender, and/or BMI) were assessed in multivariable regression models. All analyses were performed using SAS 9.4 (SAS Institute, Inc., Cary, NC).

\section{Results}

\section{Subject characteristics}

Subject characteristics are presented in Table 1. Participants were predominantly male $(88.6 \%)$, were $43.7 \pm 17.0$ (SD) years of age (ranged from 18.0 to 76.0 ) and had a mean BMI of $26.7 \pm 5.9$ (6.8-42.0). Most SCI participants had motor complete injuries (77.8\%). SCI participants were more likely to be male, were younger, had lower BMI, and had higher PAMM levels than the uninjured controls. 
Clinical and demographic factors associated with serum PAMM levels

When considering only the No SCI group in univariate analyses, we found no significant associations with PAMM levels $(\mathrm{p}=0.13-0.74$, Table 2$)$. When considering only the SCI group, PAMM levels were positively associated with age, were greater in men than in women, and were greater in motor complete compared to motor incomplete SCI. Results were similar when considering the total cohort and PAMM levels were positively associated with BMI. When considering the total cohort in multivariable models adjusting for these factors (Table 3),

\section{Table 1 Participant characteristics}

\begin{tabular}{|c|c|c|c|c|}
\hline Variable & No SCI $(n=51)$ & Acute SCI $(n=54)$ & Total cohort $(n=105)$ & $p$ \\
\hline Male, n (\%) & $41(80.0)$ & $52(96.3)$ & $93(88.6)$ & 0.01 \\
\hline Age (years) $[$ Mean $\pm S D]$ & $49.6 \pm 15.5$ & $38.1 \pm 16.6$ & $43.7 \pm 17.0$ & 0.0004 \\
\hline $\mathrm{BMI}\left(\mathrm{kg} / \mathrm{m}^{2}\right)[$ Mean $\pm \mathrm{SD}]$ & $28.8 \pm 5.1$ & $24.8 \pm 6.0$ & $26.7 \pm 5.9$ & 0.0004 \\
\hline PAMM (ng/ml) [Mean \pm SD] & $1.25 \pm 1.17$ & $2.53 \pm 1.70$ & $1.91 \pm 1.59$ & $<0.0001$ \\
\hline Motor complete, n (\%) & N/A & $42(77.8)$ & $42(40.0)$ & N/A \\
\hline
\end{tabular}

Table 2 Univariate factors associated with natural log PAMM

\begin{tabular}{|c|c|c|c|c|c|c|c|c|c|}
\hline \multirow[t]{2}{*}{ Variable } & \multicolumn{3}{|l|}{ No SCl $(n=51)$} & \multicolumn{3}{|c|}{ Acute SCI $(n=54)$} & \multicolumn{3}{|c|}{ Total cohort $(n=105)$} \\
\hline & $\beta \pm S E$ & $e^{\beta}$ & $p$ & $\beta \pm S E$ & $e^{\beta}$ & $p$ & $\beta \pm S E$ & $e^{\beta}$ & $\mathbf{p}$ \\
\hline Age (years) & $-0.008 \pm 0.006$ & 0.99 & 0.23 & $-0.02 \pm 0.007$ & 0.98 & 0.02 & $-0.02 \pm 0.005$ & 0.98 & 0.0002 \\
\hline \multirow[t]{2}{*}{ BMI $\left(\mathrm{kg} / \mathrm{m}^{2}\right)$} & $-0.03 \pm 0.02$ & 0.97 & 0.13 & $-0.009 \pm 0.02$ & 0.99 & 0.64 & $-0.04 \pm 0.01$ & 0.96 & 0.01 \\
\hline & $\begin{array}{l}\text { In PAMM } \\
(\text { mean } \pm S D)\end{array}$ & $\mathrm{e}^{\ln \text { PAMM (ng/ml) }}$ & $p$ & $\begin{array}{l}\text { In PAMM } \\
(\text { mean } \pm \text { SD })\end{array}$ & $\mathrm{e}^{\ln \text { PAMM (ng/ml) }}$ & $p$ & $\begin{array}{l}\text { In PAMM } \\
(\text { mean } \pm \text { SD })\end{array}$ & $\mathrm{e}^{\ln \text { PAMM (ng/ml) }}$ & $p$ \\
\hline \multicolumn{10}{|l|}{ Gender } \\
\hline Male & $-0.04 \pm 0.75$ & 0.96 & 0.74 & $0.70 \pm 0.80$ & 2.01 & 0.03 & $0.37 \pm 0.86$ & 1.45 & 0.03 \\
\hline Female & $-0.12 \pm 0.52$ & 0.89 & & $-0.56 \pm 1.03$ & 0.57 & & $-0.20 \pm 0.59$ & 0.82 & \\
\hline \multicolumn{10}{|l|}{$\mathrm{SCl}$ severity } \\
\hline Motor complete & N/A & & N/A & $0.76 \pm 0.78$ & 2.14 & 0.06 & $0.76 \pm 0.78$ & $2.13^{* \wedge}$, & $<0.0001$ \\
\hline Motor incomplete & & & & $0.25 \pm 0.93$ & 1.28 & & $0.25 \pm 0.93$ & $1.28^{\wedge \wedge}$ & \\
\hline $\mathrm{NoSCl}$ & & & & & & & $-0.06 \pm 0.71$ & $0.94^{* *}$ & \\
\hline
\end{tabular}

${ }^{*} \mathrm{p}<0.0001$ compared to reference group, ${ }^{* *}$ reference group, ${ }^{\wedge} \mathrm{p}=0.04$ compared to reference group, ${ }^{\wedge}$ reference group

Table 3 Multivariable factors associated with natural log PAMM

\begin{tabular}{|c|c|c|c|}
\hline \multicolumn{4}{|c|}{ Model $\mathrm{p}<0.0001, \mathrm{r}^{2}=0.26, \mathrm{n}=105$ (total cohort) } \\
\hline Variable & $\beta \pm S E$ & $e^{\beta}$ & $\mathrm{p}$ \\
\hline Age (years) & $-0.01 \pm 0.005$ & 0.99 & 0.03 \\
\hline \multirow[t]{2}{*}{$\mathrm{BMI}\left(\mathrm{kg} / \mathrm{m}^{2}\right)$} & $-0.01 \pm 0.01$ & 0.99 & 0.40 \\
\hline & Mean $\mathrm{PAMM} \pm \mathrm{SE}$ & $\mathrm{e}^{\ln \text { PAMM (ng/ml) }} \pm S E$ & \\
\hline \multicolumn{4}{|l|}{ Gender } \\
\hline Males & $0.36 \pm 0.09$ & $1.43 \pm 1.09$ & 0.23 \\
\hline Females & $0.06 \pm 0.23$ & $1.06 \pm 1.26$ & \\
\hline $\mathrm{SCl}$ severity & & & 0.01 \\
\hline Motor complete & $0.50 \pm 0.18$ & $1.65 \pm 1.20^{*}$ & \\
\hline Motor incomplete & $0.19 \pm 0.23$ & $1.21 \pm 1.26$ & \\
\hline $\mathrm{NoSCl}$ & $-0.06 \pm 0.13$ & $0.94 \pm 1.14^{* *}$ & \\
\hline
\end{tabular}

${ }^{*} \mathrm{p}=0.003$ compared to reference group, ${ }^{* *}$ reference group 
PAMM levels were no longer significantly associated with gender $(\mathrm{p}=0.23)$ or BMI $(\mathrm{p}=0.45)$. PAMM levels increased $0.99 \mathrm{ng} / \mathrm{ml}$ for every year increase in age $(\mathrm{p}=0.04)$. Participants with motor complete SCI had significantly greater PAMM levels than those with No SCI $(1.70 \mathrm{ng} / \mathrm{ml}$ versus $0.94 \mathrm{ng} / \mathrm{ml}, \mathrm{p}=0.002)$. Results were similar when considering only the SCI group $(p=0.02$ for age and $\mathrm{p}=0.04$ for gender, data not shown). We found no significant associations with PAMM levels when considering only the No SCI group $(\mathrm{p}=0.23-0.67$, data not shown) in multivariable models.

\section{Discussion}

Here we assessed factors associated with circulating PAMM levels in 54 adults with acute, traumatic SCI and in 51 adult men and women without SCI. Factors associated with PAMM levels varied based on the presence or absence of acute SCI. When adjusting for BMI, age and gender, mean PAMM levels were significantly greater in motor complete SCI compared to uninjured controls (Model $\mathrm{p}<0.0001$ ). This model explained $26 \%$ of the variation in circulating PAMM levels.

The lack of validated biomarkers of injury severity, neurological recovery, or response to therapy after spinal cord injury is considered a major limitation to both clinical care and to the development of adequately designed and powered clinical trials. The variability in spontaneous neurologic recovery at 1-year post-injury is large and neurological examination does not differentiate between the estimated $30 \%$ who experience recovery from those who do not. This is considered a serious limitation to both clinical care and to the development of adequately designed and powered clinical trials. Identification of a novel prognostic biomarker of spontaneous recovery would make clinical trial recruitment more economical and efficient as well as accelerate neuro-recovery research. These results suggest that PAMM may be a biomarker of injury severity in acute SCI. PAMM may also be a novel prognostic biomarker of neurological recovery in acute SCI. That is, PAMM levels tested within the first 3 months after injury might be used to identify those who will experience neurological recovery from those who will not. This hypothesis should be tested in future studies.

We previously reported that PAMM is highly expressed in both white and brown adipose tissue and its expression increases in obesity [8]. Adipocytes are well-known protein secretors, particularly of hormone-like adipokines, which affect several physiological processes, including energy balance and insulin sensitivity $[9,10]$. The endoplasmic reticulum (ER) membrane-located PAMM plays a key role in adipocyte biology, notably assembly and secretion of disulfide-bond containing proteins, including adiponectin and collagen isoforms. PAMM is secreted from mature human adipocytes (but not preadipocytes), has anti-inflammatory properties, and suppresses macrophage activation by inhibiting MAPK signaling pathway. PAMM exerts its biological activity by inhibiting signal transduction pathways. However, the PAMM response to various stimuli varies, possibly due to the involvement of several different signaling pathways [11]. Regardless of the signal transduction pathways involved, the mechanism of PAMM action is dependent, at least in part, upon its anti-redox activity. For example, PAMM negatively regulates cytokine-induced inflammatory responses in vascular endothelial cells by suppressing MAPK and NF-kB signal pathways. This suppression was shown to be partially dependent upon the antiredox activity of PAMM [11]. Not only does PAMM inhibit responses of macrophages and endothelial cells to inflammatory stimuli, but its expression in human atheromatous tissue was nearly 3 -fold as compared to normal, reflecting its potential as a therapeutic anti-inflammatory agent for the treatment of vascular inflammatory diseases [11]. The anti-redox activity of PAMM likely also inhibits TNF $\alpha$-induced MAPK and NF-kB activation through a reduction in ER stress by promoting protein folding and secretion [11]. We recently reported that mutations in the CXXC motif of PAMM suppress its anti-redox activity without suppressing the production of inflammatory cytokines in lipopolysaccharide-stimulated macrophages, indicating that PAMM's anti-inflammatory and anti-oxidant properties are independent [8].

Mediators of cellular and subcellular oxidative stress have received considerable attention as potential novel neuroprotective targets in acute $\mathrm{SCI}$, including the involved genes, proteins and factors [2, 4, 8, 12]. PAMM is a promising candidate biomarker for SCI because of its dual independent anti-inflammatory and anti-oxidant properties, and because SCI is characterized by both oxidative stress and inflammatory reaction [13-15]. Several other putative inflammatory biomarkers of SCI include interleukins, TGF-B1, insulin-like growth factor 1 (IGF-1) and soluble CD95 ligand (sCD95L). But, there are conflicting reports in the literature about the utility of each as a predictor of inflammatory-related pathologies in SCI (see for a review, [5]). To ensure accuracy and repeatability, these markers have been suggested to be used in parallel with other diagnostic biomarkers for SCI [5]. Additional work is needed to validate these candidate biomarkers prior to implementing use in clinical care.

In this study we report that PAMM is a candidate circulating biomarker of acute, traumatic SCI. We hypothesize that adipose tissue is the source of circulating PAMM. It is unclear why associations between age and gender are stronger in the SCI group compared to uninjured 
controls, or the mechanisms leading to increased circulating PAMM levels after traumatic SCI. It is possible that increased PAMM expression is an innate native anti-inflammatory response to acute neurotrauma. Also, it is possible that a larger study is required to assess associations between PAMM and clinical or demographic factors, such as age, BMI, or gender, in the general population. Further work is needed to test this hypothesis. Strengths of the current study include the use of a reliable PAMM assay and identification of potential confounding factors, including age. Study limitations include a relatively small sample size with few women. The association between gender and PAMM in acute SCI should be confirmed in a larger study with more women. Despite these limitations, we conclude that PAMM has potential as a novel biomarker of acute SCI. Future research is needed to confirm and validate these findings.

\section{Conclusions}

Our results suggest that PAMM may be a novel biomarker of neurological injury or of native anti-inflammatory responses to neurological injury. More work is needed to establish the role of PAMM and other adipocyte-derived factors in the acute response to neurotrauma.

\section{Acknowledgements}

Not applicable.

\section{Authors' contributions}

Study design: LM, RB. Study conduct: LM, RB, YX, NN, PKJ. Data collection: YX NN. Data analysis: LM, RB, XY, NN, PKJ. Data interpretation: LM, RB, PKJ. Drafting manuscript: $L M, R B, Y X, N N$, PKJ. Revising manuscript content: $L M, R B, Y X, N N$, PKJ. Approving final version of manuscript: LM, RB, YX, NN, PKJ. All authors take responsibility for the integrity of the data analysis. All authors read and approved the final manuscript.

\section{Funding}

This study received support from: the National Institute of Arthritis and Musculoskeletal and Skin Diseases [1R01AR059270-01].

\section{Availability of data and materials}

The datasets used and/or analysed during the current study are available from the corresponding author on reasonable request.

\section{Ethics approval and consent to participate}

Our Institutional Review Board approved this study.

\section{Consent for publication}

Not applicable.

\section{Competing interests}

The authors declare that they have no competing interests.
Received: 13 December 2019 Accepted: 14 March 2020

Published online: 24 March 2020

\section{References}

1. Lee BB, Cripps RA, Fitzharris M, Wing PC. The global map for traumatic spinal cord injury epidemiology: update 2011, global incidence rate. Spinal Cord. 2014;52:110-6.

2. Ydens E, Palmers I, Hendrix S, Somers V. The next generation of biomarker research in spinal cord injury. Mol Neurobiol. 2017:54:1482-99.

3. Visavadiya NP, Patel SP, VanRooyen JL, Sullivan PG, Rabchevsky AG. CelIular and subcellular oxidative stress parameters following severe spinal cord injury. Redox Biol. 2016;8:59-67.

4. Xu L, Botchway BOA, Zhang S, Zhou J, Liu X. Inhibition of NF-kappaB signaling pathway by resveratrol improves spinal cord injury. Front Neurosci. 2018;12:690.

5. Albayar AA, Roche A, Swiatkowski P, Antar S, Ouda N, Emara E, Smith DH, Ozturk AK, Awad BI. Biomarkers in spinal cord injury: prognostic insights and future potentials. Front Neurol. 2019;10:27.

6. Xu Y, Morse LR, da Silva RAB, Wang D, Battaglino RA. A short report: PAMM, a novel antioxidant protein, induced by oxidative stress. Redox Biol. 2015;6:446-53.

7. Xu Y, Morse LR, da Silva RA, Odgren PR, Sasaki H, Stashenko P, Battaglino RA. PAMM: a redox regulatory protein that modulates osteoclast differentiation. Antioxid Redox Signal. 2010;13:27-37.

8. Guo F, He H, Fu ZC, Huang S, Chen T, Papasian CJ, Morse LR, Xu Y, Battaglino RA, Yang XF, et al. Adipocyte-derived PAMM suppresses macrophage inflammation by inhibiting MAPK signalling. Biochem J. 2015;472:309-18.

9. Fasshauer M, Bluher M. Adipokines in health and disease. Trends Pharmacol Sci. 2015;36:461-70.

10. Jedrychowski MP, Liu L, Laflamme CJ, Karastergiou K, Meshulam T, Ding SY, Wu Y, Lee MJ, Gygi SP, Fried SK, Pilch PF. Adiporedoxin, an upstream regulator of ER oxidative folding and protein secretion in adipocytes. Mol Metab. 2015:4:758-70.

11. He H, Guo F, Li Y, Saaoud F, Kimmis BD, Sandhu J, Fan M, Maulik D, Lessner S, Papasian CJ, et al. Adiporedoxin suppresses endothelial activation via inhibiting MAPK and NF-kappaB signaling. Sci Rep. 2016;6:38975.

12. Gorgey AS, Wells KM, Austin TL. Adiposity and spinal cord injury. World J Orthop. 2015;6:567-76.

13. Hart JE, Morse L, Tun CG, Brown R, Garshick E. Cross-sectional associations of pulmonary function with systemic inflammation and oxidative stress in individuals with chronic spinal cord injury. J Spinal Cord Med. 2016:39:344-52.

14. Zhu Y, Xu Q, Sha WP, Zhao KP, Wang LM. MiR-219-5p promotes spinal cord injury recovery by inhibiting NEUROD2-regulated inflammation and oxidative stress. Eur Rev Med Pharmacol Sci. 2019;23:37-43.

15. Pourkhodadad S, Oryan SH, Kaka G, Sadraie SH. Neuroprotective effects of combined treatment with minocycline and olfactory ensheathing cells transplantation against inflammation and oxidative stress after spinal cord injury. Cell J. 2019;21:220-8.

\section{Publisher's Note}

Springer Nature remains neutral with regard to jurisdictional claims in published maps and institutional affiliations. 